\title{
Intensity Distortion Induced by Cross-Phase Modulation and Chromatic Dispersion in Optical- Fiber Transmissions with Dispersion Compensation
}

\author{
Giovanni Bellotti, Student Member, IEEE, Matteo Varani, Student Member, IEEE, \\ Cristian Francia, Student Member, IEEE, and Alberto Bononi, Member, IEEE
}

\begin{abstract}
In dispersion compensated systems, the intensity distortion induced by the interplay between cross-phase modulation and fiber chromatic dispersion can be a primary cause of transmission degradation. This interplay is mostly studied by time-consuming computer simulations. This letter introduces a new model of this interplay in fiber transmissions with dispersion compensation, leading to a linear filter that, applied to the input intensity of a modulated interfering channel, gives the intensity distortion of a continuous-wave probe signal at the receiver. The model can be of significant value in the search for optimized dispersion maps.
\end{abstract}

Index Terms - Crosstalk, dispersion compensation, intensity modulation, optical fiber nonlinearity, relative intensity noise, wavelength-division multiplexing.

\section{INTRODUCTION}

D ISPERSION compensation in long-distance high-speed wavelength-division-multiplexing (WDM) transmission systems should ideally suppress the linear distortion due to group velocity dispersion (GVD) while strongly reducing fourwave mixing effects [1]. However, self-phase modulation (SPM) and cross-phase modulation (XPM), interacting with GVD, can still be primary causes of transmission degradation.

This letter concentrates on XPM. While the phase-tointensity (PM/IM) conversion induced through GVD [2] by a phase modulation present at the input of the fiber can be perfectly undone by a compensating fiber, the one induced by XPM components generated away from the input cannot be perfectly compensated, and the resulting residual intensity distortion impairs the performance of intensity modulated, direct detected systems. Such intensity distortion has been mostly studied by means of computer simulations, because the nonlinear Schrödinger equation is not analytically solvable for general pulses when both the nonlinear and the dispersive terms are present [3]. Simulations involving XPM are almost prohibitively time consuming, because they require the propagation of all the WDM channels.

This letter introduces a new linear model based on the one in [2] leading to a filter that, applied to the input intensities of the

Manuscript received March 31, 1998; revised September 3, 1998. This work was supported in part by the European Community under INCO-DC Project 950959 "DAWRON," and in part by a CSELT grant.

The authors are with the Dipartimento di Ingegneria dell'Informazione, Università di Parma, I-43100 Parma, Italy.

Publisher Item Identifier S 1041-1135(98)08777-1. modulated interfering channels, gives the intensity distortion of a continuous-wave (CW) probe signal at the end of a compensated system. The key idea is the following. The XPM at the end of the link is the sum of the XPM contributions generated in each infinitesimal fiber segment. Each infinitesimal XPM contribution gives an infinitesimal intensity distortion by PM/IM conversion [2] at the end of the link. The linear model postulates that such infinitesimal contributions add up to give the total output intensity distortion. The range of applicability of the model is roughly the same as that in [2]. The model can be of significant value in the search for optimized dispersion maps.

The paper is organized as follows. Section II derives the XPM intensity filters, both for a single fiber and for a compensated system composed of several spans. Section III compares theory and simulation to validate the model for typical spanby-span compensation schemes. Finally, Section IV concludes the paper.

\section{THEORY}

Consider one span of single-mode fiber (SMF) of length $L$, with two copropagating channels, $s$ and $p$, having the same polarization. Probe channel $s$ is $\mathrm{CW}$, while pump channel $p$ is intensity modulated, being $P_{p}(0, \omega)$ the Fourier transform of its power at the beginning of the fiber. Let $v_{s}$ and $v_{p}$ be the group velocities of the two channels, and let $d_{s p} \triangleq 1 / v_{s}-$ $1 / v_{p} \cong D \Delta \lambda_{s p}$ be the walkoff parameter [3], with $D$ the fiber dispersion at the probe wavelength and $\Delta \lambda_{s p}$ the channel spacing.

The pump power at coordinate $z$ along the fiber, in the assumption of undistorted pump, has Fourier transform (with respect to a time frame moving with the probe group velocity) given by $P_{p}(z, \omega) \triangleq P_{p}(0, \omega) \exp \left(\left(-\alpha+j \omega d_{s p}\right) z\right)$. The imaginary argument of the exponential term accounts for the time shift due to channel walkoff. The probe phase induced at $z$ through XPM by propagation of such pump over an infinitesimal segment $d z$ is $d \theta_{s p}(z, \omega)=-2 \gamma P_{p}(z, \omega) d z$. Such phase modulation enters the remaining $L-z \mathrm{~km}$ of fiber: if such fiber were purely linear, it would produce at its output a relative probe power distortion [2]

$$
\frac{d P_{s p}(z, \omega)}{\left\langle P_{s}\right\rangle}=-2 \sin \left[\omega^{2} \frac{\lambda^{2}}{4 \pi c} D(L-z)\right] d \theta_{s p}(z, \omega)
$$


where $\left\langle P_{s}\right\rangle$ is the time averaged output probe power, $\lambda$ the probe wavelength and $c$ the light velocity. The SPM induced by such infinitesimal power distortion is neglected. If the infinitesimal intensity contributions add up, the total relative output power distortion on the probe is obtained by integrating (1) in $d z$ over the fiber length:

$$
\frac{\Delta P_{s p}(\omega)}{\left\langle P_{s}\right\rangle}=P_{p}(0, \omega) H_{s p}(\omega)
$$

where we defined the XPM/IM filter as

$$
\begin{array}{r}
H_{s p}(\omega) \\
\triangleq 4 \gamma \int_{0}^{L} \exp \left(\left(-\alpha+j \omega d_{s p}\right) z\right) \sin \left[\omega^{2} \frac{\lambda^{2}}{4 \pi c} D(L-z)\right] d z \\
\left.=-2 \gamma j\left\{\begin{array}{l}
\exp \left(j\left(\lambda^{2} / 4 \pi c\right) D_{r} \omega^{2}\right) \\
\cdot\left(\frac{1-\exp \left(\left(\left(-\alpha+j\left(d_{s p} \omega-\left(\lambda^{2} / 4 \pi c\right) D \omega^{2}\right)\right) L\right)\right.}{\alpha-j\left(d_{s p} \omega-\frac{\lambda^{2}}{4 \pi c} D \omega^{2}\right)}\right) \\
-\exp \left(-j\left(\lambda^{2} / 4 \pi c\right) D_{r} \omega^{2}\right)
\end{array}\right)\right\} \\
\left.\cdot\left(\frac{\left.1-\exp \left(-\alpha+j\left(d_{s p} \omega+\left(\lambda^{2} / 4 \pi c\right) D \omega^{2}\right)\right) L\right)}{\alpha-j\left(d_{s p} \omega+\frac{\lambda^{2}}{4 \pi c} D \omega^{2}\right)}\right)\right\}
\end{array}
$$

where $D_{r} \triangleq D L$ is the residual accumulated dispersion from the beginning of the fiber to the end of the system.

Now consider the general case of a chain of $M$ endamplified fiber links, with $\alpha_{i}, \gamma_{i}, D_{i}, l_{i}, d_{s p}^{(i)}$ the attenuation, nonlinear and dispersion coefficients, the length and the walkoff parameter of the $i$ th link, $i=1, \cdots, M$, respectively. Suppose that the $i$ th amplifier has gain $G_{p}^{(i)}$ for the pump, so that the pump power at the input of the $k$ th link, in the undistorted pump assumption, is $P_{p}\left(L_{k}, \omega\right)=C_{p}^{(k)} P_{p}(0, \omega)$, where $L_{k} \triangleq \sum_{i=1}^{k-1} l_{i}$ and $C_{p}^{(k)} \triangleq \Pi_{i=1}^{k-1} \exp \left(\left(-\alpha_{i}+j \omega d_{s p}^{(i)}\right) l_{i}\right) G_{p}^{(i)}$, with $C_{p}^{(1)} \triangleq 1$. The residual accumulated dispersion from the beginning of the $k$ th link to the end of the system is $D_{r}^{(k)} \triangleq \sum_{i=k}^{M} D_{i} l_{i}$. Reasoning as before, the XPM contribution $d \theta_{s p}^{(k)}(z, \omega)=-2 \gamma_{k} P_{p}\left(L_{k}, \omega\right) \exp \left(\left(-\alpha_{k}+j \omega d_{s p}^{(k)}\right) z\right) d z$ generated at coordinate $z$ of the $k$ th fiber enters a "purely linear equivalent fiber" so that its contribution to the relative output power distortion on the probe is $\left(d P_{s p}^{(k)}(z, \omega) /\left\langle P_{s}\right\rangle\right)=$ $-2 \sin \left[\omega^{2}\left(\lambda^{2} / 4 \pi c\right)\left(D_{r}^{(k)}-D_{k} z\right)\right] d \theta_{s p}^{(k)}(z, \omega)$. Integrating as before over the $k$ th fiber length and adding the contributions of all fiber segments, the overall XPM/IM filter for the $M$ links becomes

$$
H_{s p}(\omega)=\sum_{k=1}^{M} C_{p}^{(k)} H_{s p}^{(k)}(\omega)
$$

where $H_{s p}^{(k)}(\omega)$ is given by (3) with the appropriate parameters of the $k$ th fiber. The relative probe power distortion at the system end is given again by (2). When several pump channels
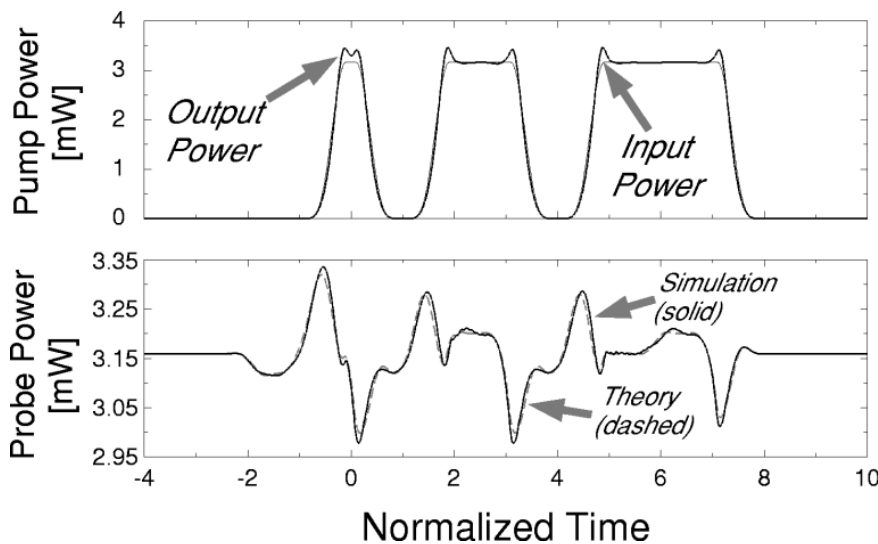

Fig. 1. Simulations of a NZDF + SMF system, $R=10 \mathrm{~Gb} / \mathrm{s}, M=10$ spans, $\Delta \lambda=0.8 \mathrm{~nm}$. Top row: Input and output pump power $(\mathrm{mW})$. Bottom row: Output probe power. Time is normalized to the bit time 1/R.

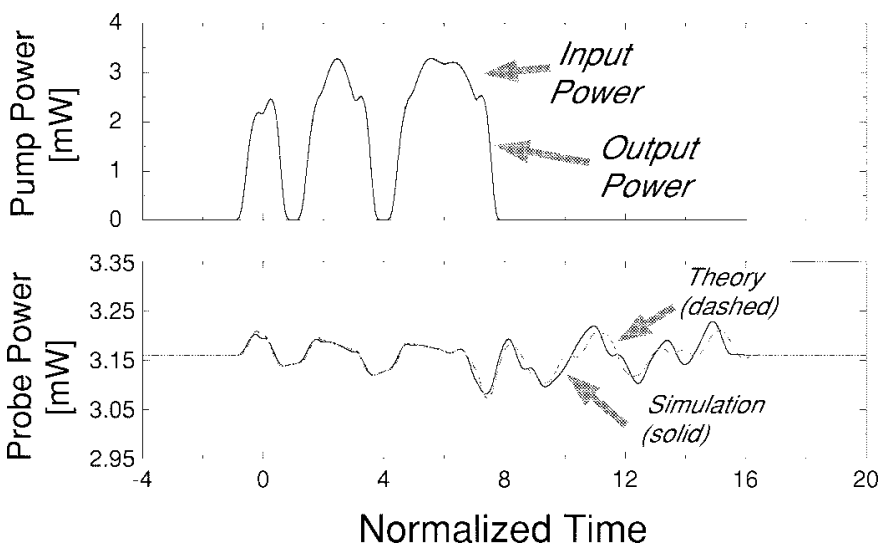

Fig. 2. Simulations of a SMF + DCF system, $R=10 \mathrm{~Gb} / \mathrm{s}, M=10$ spans, $\Delta \lambda=0.8 \mathrm{~nm}$. Top row: Input and output pump power [mW]. Bottom row: Output probe power. Time is normalized to the bit time $1 / \mathrm{R}$.

are present, the total relative probe power distortion can be written as the sum of the contributions due to each pump.

\section{Simulations}

In Figs. 1 and 2, we compare the results of simulations performed by the split-step Fourier method [3] with the predictions of (2). Simulations include the effects of GVD, SPM and XPM for the pump, and GVD and XPM for the probe. Simulations are carried out for a 10 span WDM system, perfectly compensated after each span, with $5 \mathrm{dBm}$ peak power for both pump and probe and channel spacing $\Delta \lambda=0.8 \mathrm{~nm}$. The pump is on-off keying (OOK) modulated with nonreturnto-zero (NRZ) raised cosine pulses (rolloff 0.8 ) at a bit rate $R=10 \mathrm{~Gb} / \mathrm{s}$, while the probe is $\mathrm{CW}$. Fig. 1 refers to a system in which the transmission fiber for each span is a nonzero dispersion fiber (NZDF), with dispersion $D=-2$ $\mathrm{ps} / \mathrm{nm} / \mathrm{km}$, length $l=85 \mathrm{~km}$, and a standard SMF with $D=17 \mathrm{ps} / \mathrm{nm} / \mathrm{km}$ and length $l=10 \mathrm{~km}$ is used for span compensation. Other parameters common to the two fibers are the dispersion slope $D^{\prime}=0.07 \mathrm{ps} / \mathrm{km} / \mathrm{nm}^{2}$, the nonlinear coefficient $\gamma=2.35 \mathrm{~W}^{-1} \cdot \mathrm{km}^{-1}$ and the attenuation $\alpha=0.21$ $\mathrm{dB} / \mathrm{km}$. Fig. 2 refers to a system in which the transmission fiber is a SMF as before, with $l=57 \mathrm{~km}$, and a dispersion 


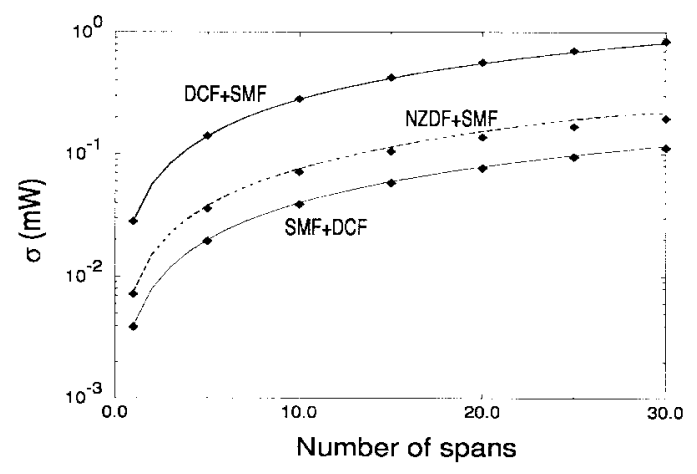

Fig. 3. Probe power standard deviation $\sigma$ versus number of spans in three different maps. Solid: Simulation. Diamonds: Theory. Two neighboring pumps at $\Delta \lambda= \pm 0.8 \mathrm{~nm}$, OOK modulated at $10 \mathrm{~Gb} / \mathrm{s}$. Peak power of $5 \mathrm{dBm} / \mathrm{ch}$.

compensating fiber (DCF) with $D=-95 \mathrm{ps} / \mathrm{nm} / \mathrm{km}, l=10.2$ $\mathrm{km}, D^{\prime}=0.07 \mathrm{ps} / \mathrm{km} / \mathrm{nm}^{2}, \gamma=6 \mathrm{~W}^{-1} \cdot \mathrm{km}^{-1}, \alpha=0.6 \mathrm{~dB} / \mathrm{km}$ is used for compensation. In both figures, the wavelength of exact compensation is halfway between channels. The figures show the power of the pump both at the input and at the output of the whole link (top), and the output power of the probe (bottom), both simulated and predicted by (2). The pattern "10 110 111" is visible in the pump intensity. As seen from the figures, the linear model predicts reasonably well the shape of the output probe power (bottom graphs), even when the hypothesis of undistorted pump is violated (top graphs). This is due to the lowpass filtering action of walkoff on the generation of XPM [4]. We note that the probe modulation is larger in the NZDF + SMF system, because the XPM generation is more efficient than in the SMF + DCF system, and thus the intensity distortion is larger, as per (1).

In Fig. 3, we consider the XPM-induced power modulation caused by two neighboring pumps on a central $\mathrm{CW}$ probe. The pumps are OOK modulated at $10 \mathrm{~Gb} / \mathrm{s}$, with independent patterns of 128 bits. We define the sample variance as $\sigma^{2}=$ $(1 / N) \sum_{k=1}^{N}\left[P_{s}\left(k\left(T_{0} / N\right)\right)-\left\langle P_{s}\right\rangle\right]^{2}$, where $P_{s}$ is the probe power and $N$ is the number of samples in the observation window of 128 bits. The figure plots $\sigma$ versus the number of compensated spans, both for simulations and for (2). If the XPM induced intensity noise on ONE's and ZERO's is computed, to a first approximation, as if the probe pulse had an average constant value, then, in the presence of amplified spontaneous emission noise, the error probability can be evaluated by a standard Gaussian approximation, and thus depends directly on the parameter $\sigma$ relative to the ONE and ZERO power levels. Such Gaussian approximation is sufficient for comparisons among compensating schemes and in the search for optimal maps. If a more precise performance evaluation is needed [5], the filter in (3) can be used to estimate the actual non-Gaussian statistics of the XPM-induced intensity noise. The parameter $\sigma$ in Fig. 3 refers to a CW probe corresponding to the ONE power level, and three compensating schemes are compared: the NZDF + SMF and SMF + DCF are the maps leading to Figs. 1 and 2; the third, DCF + SMF, uses the same fibers as the SMF + DCF, but the compensating fiber is placed right after the amplifier. We see that the system minimizing the XPM-induced intensity noise is the SMF + DCF, while the more common inverse map DCF + SMF, which optimizes the effect of SPM [6], performs worse in terms of XPM-induced intensity noise because of the large, uncompensated XPM generated at the beginning of the SMF fiber, where the power level is still high.

\section{CONCLUSION}

In this letter, we introduced a new linear model for the XPM-induced intensity distortion on a $\mathrm{CW}$ probe in a dispersion compensated transmission system. By comparison to simulations we have shown that the model well captures the essence of the interaction between XPM and GVD, giving accurate predictions within the applicability range of the model in [2] upon which it is based, and within the validity of the undistorted probe assumption. The model neglects XPMinduced SPM effects on the probe. It is possible to extend the present model to include the SPM generated by a modulated probe [7]. As long as the intensity distortions due to SPM and XPM can be considered as additive and independent, the system performance evaluation greatly simplifies, and XPM-induced intensity modulation can be treated as additive intensity noise. We believe the model, together with its extensions to SPM, can be of great value in both quickly evaluating system performance and in the search for optimized dispersion maps.

\section{REFERENCES}

[1] A. R. Chraplyvy, A. H. Gnauck, R. W. Tkach, R. M. Derosier, C. R. Giles, B. M. Nyman, G. A. Freguson, J. W. Sulhoff, and J. L. Zyskind, "One-third terabit/s transmission through $150 \mathrm{~km}$ of dispersion-managed fiber," IEEE Photon. Technol. Lett., vol. 7, pp. 98-100, Jan. 1995.

[2] J. Wang and K. Petermann, "Small signal analysis for dispersive optical fiber communication systems," J. Lightwave Technol., vol. 10, pp. 96-100, Jan. 1992.

[3] G. P. Agrawal, Nonlinear Fiber Optics, 2nd ed. New York: Academic, 1995, ch. 7.

[4] G. Bellotti, M. Varani, C. Francia, and A. Bononi, "Intensity/crossphase/intensity conversion filters in dispersion compensated multiwavelength transmission systems," presented at the 1998 Conf. Information Science and Systems, Princeton, NJ, Mar. 1998, paper TP4.2.

[5] J. J. O'Reilly and J. R. F. da Rocha, "Improved error probability evaluation methods for direct detection optical communication systems," IEEE Trans. Inform. Theory, vol. 33, pp. 839-848, Nov. 1987.

[6] D. M. Rothnie and J. E. Midwinter, "Improved standard fiber performance by positioning the dispersion compensating fiber," Electron. Lett., vol. 32, no. 20, pp. 1907-1908, Sept. 1996

[7] M. Varani, "Analisi degli effetti di self e cross-phase modulation in sistemi multicanale con compensazione della dispersione cromatica," Laurea thesis, Univ. di Parma, Parma, Italy, Apr. 1998. 